pathway decreases neuronal injury.

In a separate study, the same group found that similar mechanisms explain why engineered human vascular tissue constricts when subjected to force. The pathway could be a target for preventive drugs, the authors say.

PLoS ONE 6, e22899 (2011);

Proc. Natl Acad. Sci. USA

http://dx.doi.org/10.1073/

pnas.1105860108(2011)

\section{MATERIALS \\ Soft optics from organic gels}

Optical devices such as lenses and prisms can be made from organic gels developed by Kana Sureshan and his colleagues at the Indian Institute of Science Education and Research in Thiruvananthapuram.

They used very low concentrations of two different molecules based on the sixcarbon carbohydrate mannitol to make a series of gels from hydrocarbon-based solvents such as paraffin oil and pump oil. Shapes formed from these gels were transparent and remained stable for months. The gels could filter ultraviolet light and bend visible light much like glass does. Moreover, optical devices made from the gels were shatter- and scratchproof, thanks to the gels' ability to fuse together after being cut into pieces.

Angew. Chem. Int. Edn

http://dx.doi.org/10.1002/

anie.201103584 (2011)

\section{ADDICTION}

\section{Target for blocking cocaine}

The effects of cocaine can be counteracted in mice by stimulating an enigmatic brain receptor, suggesting a possible target for addiction treatment.

The $\mathrm{CB}_{1}$ receptor in the brain has been implicated in drug addiction, but the role of the $\mathrm{CB}_{2}$ receptor has not been clear. So Zheng-Xiong Xi and his team at the National Institute on Drug Abuse in Baltimore,

Maryland, gave cocaineaddicted mice a compound that stimulates the $\mathrm{CB}_{2}$ receptor. The animals self-administered cocaine less frequently, whereas no effect was seen in mice lacking the $\mathrm{CB}_{2}$ receptor.

The effect occurred after intranasal but not intravenous injection of the compound, suggesting that it involves $\mathrm{CB}_{2}$ receptors in the brain but not in other parts of the body. Nature Neurosci. http://dx.doi. org/10.1038/nn.2874 (2011)

\title{
ARCHAEOLOGY
}

\section{Another origin of domestic dogs}

The discovery of ancient dog bones in France point to a European origin for man's best friend.

Humans domesticated dogs from wild wolves, and genetic and archaeological studies have indicated that domestication could have occurred in East Asia and the Middle East, as well as in Europe. Maud Pionnier-Capitan at the National Museum of Natural History in Paris and her team uncovered dozens of remains of small dogs (pictured) at two sites in southern France and another near Paris. The fossils are 11,500-15,500 years old, making them contemporaries of much larger ancient dogs found in Russia. This indicates that dog domestication may have occurred independently in various parts of Europe and Asia.

Furthermore, some of the bones bore cut marks, suggesting that ancient humans ate some of the canines.

J. Archaeol. Sci. 38, 2123-2140 (2011)

\section{CELL BIOLOGY}

\section{Microtubules beat in sync}

Certain cells bear tiny hair-like structures called cilia, which beat in wave-like patterns. The

COMMUNITY CHOICE

The most viewed papers in science

GENOMICS

\section{Customer data aid Parkinson's study}

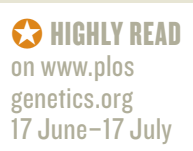

Data collected by a personal genetic testing company in California reveal new associations between two genomic areas and a risk of Parkinson's disease.

Chuong Do and Nicholas Eriksson at 23andMe in Mountain View and their colleagues compared the genomic sequences of more than 3,400 people with Parkinson's disease with those from 29,000 23andMe customers who reported not having the disease in an e-mail questionnaire. The authors confirmed 20 previous genetic associations with the disease in addition to revealing the two new links. However, they suggest that many more genetic associations with the disease remain to be found. PLoS Genet. 7, e1002141 (2011) behaviour is unknown, but researchers have succeeded in mimicking it with bundles of protein filaments. Zvonimir Dogic and his colleagues at Brandeis University in Waltham, Massachusetts, built the in vitro system from just three components. They used microtubules (MTs), the filaments that form the backbone of cilia; kinesin motors, the protein complexes that bind to and slide along MTs; and polyethylene glycol, which bundles the MTs together. The authors found that their bundles, when anchored at one end, beat much less frequently than cilia - which have more than 650 proteins - but synchronize their beating at high density.

The authors suggest that the sliding kinesin motors, which were engineered to bind to two adjacent MTs, move these MTs in opposite directions, resulting in the beating motion.

Science 333, 456-459 (2011) mechanism underlying this

\section{MICROBIOLOGY}

\section{Bacteria whip} around corners

By using their hair-like projections as a slingshot, bacteria can quickly swerve.

Pseudomonas aeruginosa

bacteria clump together to form biofilms during pneumonia infection and rely on small appendages called pili to attach to surfaces and pull themselves forwards. Gerard Wong at the University of California, Los Angeles, and his group used video microscopy to track the trajectories of the leading and trailing poles of $P$. aeruginosa cells. They propose that the bacteria can also use the pili to change direction. The rapid release of a single tethered pilus provides enough force to spin a bacterium around.

The authors think that this fast slingshot action helps the microbes to move through the viscous maze of polysaccharides they secrete when forming biofilms.

Proc. Natl Acad. Sci. USA http://dx.doi.org/10.1073/ pnas.1105073108(2011)

\section{DNATURE,COM}

For the latest research published by Naturevisit:

www.nature.com/latestresearch 